8

\title{
Effects of Supplementation with Curcumin on Serum Adipokine Concentrations: A Randomized Controlled Trial
}

\author{
Yunes Panahi, ${ }^{1}$ Mahboobeh Sadat Hosseini, ${ }^{2}$ Nahid Khalili, ${ }^{2}$ Effat Naimi, ${ }^{2}$ Sara Saffar \\ Soflaei, ${ }^{3}$ Muhammed Majeed, ${ }^{4}$ Amirhossein Sahebkar ${ }^{5,6^{*}}$ \\ ${ }^{1}$ Chemical Injuries Research Center, Baqiyatallah university of Medical Sciences, Tehran, Iran \\ ${ }^{2}$ Department of Endocrinology, Baqiyatallah university of Medical Sciences, Tehran, Iran \\ ${ }^{4}$ Neurogenic Inflammation Research Center, Mashhad University of Medical Sciences, Mashhad, \\ ${ }^{4}$ Sabinsa Inc, Princeton, NJ, United States \\ ${ }^{5}$ Biotechnology Research Center, Mashhad University of Medical Sciences, Mashhad, Iran \\ ${ }^{6}$ Metabolic Research Centre, Royal Perth Hospital, School of Medicine and Pharmacology, \\ University of Western Australia, Perth, Australia
} Iran

Please address all correspondence to: Amirhossein Sahebkar, Pharm.D, Ph.D, Department of Medical Biotechnology, School of Medicine, Mashhad University of Medical Sciences, Mashhad, Iran, P.O. Box: 91779-48564, Iran. Tel : 985118002288; Fax: 985118002287; E-mail:

sahebkara@mums.ac.ir; amir_saheb2000@yahoo.com

Running title: Antioxidant and Anti-inflammatory Effects of Curcuminoids

Word count: 3115

Number of figures: 2

Number of tables: 3 


\section{Abstract}

41 Background: Previous experimental studies have suggested curcumin as a safe phytochemical that can improve insulin resistance through effects on adiponectin and leptin. This study aimed to investigate the effect of curcumin on circulating adiponectin and leptin concentrations in patients

44 with metabolic syndrome (MetS).

Methods: In this pilot randomized double-blind placebo-controlled trial, subjects who met the criteria of MetS according to the National Cholesterol Education Program Adult Treatment Panel III (NCEP-ATP III) criteria were randomly assigned to curcumin ( $\mathrm{n}=59 ; 1000 \mathrm{mg} /$ day) or placebo $(n=58)$ for 8 weeks. Serum adiponectin and leptin concentrations were determined before and after intervention. The pooled effect size for the impact of curcumin supplementation on serum adiponectin and leptin levels was also estimated using random-effects meta-analysis. Results: Eight-week supplementation with curcumin was associated with a significant increase in serum adiponectin levels $(p<0.001)$, and a reduction in serum leptin concentrations $(p<0.001)$.

53 Serum leptin:adiponectin ratio was also improved by curcumin $(p<0.001)$. These beneficial effects of curcumin remained significant after adjustment for changes in serum lipids and glucose concentrations, and baseline differences in BMI and serum levels of glucose and HbA1c as potential confounders of treatment response. Meta-analysis suggested that curcumin supplementation can increase adiponectin levels by 76.78\% (95\% CI: 6.14, 147.42; $p=0.0330$ ), and reduce leptin by $26.49 \%$ (95\% CI: -70.44, 17.46), however, this latter effect size did not reach statistical significance $(p=0.238)$.

60 Conclusion: Curcumin can improve serum levels of adiponectin and leptin in patients with metabolic syndrome. This trial was registered at the UMIN Clinical Trials Registry

62 (http://www.umin.ac.jp/ctr/) under Trial No. UMIN000018339. 


\section{Abbreviations}

73 MetS: metabolic syndrome; LDL: low-density lipoprotein; HDL: high-density lipoprotein; 74 NCEP-ATP III: National Cholesterol Education Program Adult Treatment Panel III; BMI: body 75 mass index; SD: standard deviation; DBP: diastolic blood pressure; SBP: systolic blood pressure; 76 HbA1c: hemoglobin A1c; Lp(a): lipoprotein(a); hs-CRP: high-sensitivity C-reactive protein; CI: 77 confidence interval; RCT: randomized clinical trial; JNK: c-Jun N-terminal kinases; NFқB: 78 nuclear factor kappa-light-chain-enhancer of activated B cells; AMPK: 5’AMP-activated protein 79 kinase; HOMA-IR: homeostatic model assessment of insulin resistance; QUICKI: quantitative 80 insulin sensitivity check index

81

82

83

84

85

86

87

88

89

90

91

92

93

94

95

96

97

98

99

100

101 


\section{Introduction}

Metabolic syndrome (MetS), also known as syndrome $\mathrm{X}$, is a cluster of several cardiometabolic risk factors including abdominal adiposity, hyperglycemia, hypertriglyceridemia, low HDL-C and hypertension $(1,2)$. MetS prevalence ranges between 10 to $84 \%$ worldwide. According to Azimi-Nezhad et al., 55.0\% of Iranian females and 30.1\% of Iranian males have Mets (3). Insulin resistance and visceral adiposity are the key factors underlying MetS pathophysiology. Visceral adipose tissue acts as an endocrine organ and releases different kinds of cytokines named adipokines. These adipokines mediate multiple processes such as insulin sensitivity, oxidative stress and inflammation; hence their imbalance could contribute to the development of type 2 diabetes mellitus and atherosclerosis.

Adiponectin and leptin are the most studied adipokines, and their levels are known to be altered in patients with MetS. Adiponectin is a cardioprotective adipokine with anti-inflammatory properties that improve lipid and glucose metabolism, increase insulin sensitivity (4) and prevent atherogenesis (5). Several observational studies have reported an inverse association between circulating adiponectin concentrations and body weight, total cholesterol, triglycerides, blood pressure and insulin resistance, and a positive association with HDL-cholesterol (HDL-C) levels $(6,7)$. Leptin is another adipokine with a pivotal role in the regulation of energy balance in the body (8). Plasma concentrations of leptin increase with adiposity and correlate with insulin resistance $(9,10)$. Elevated plasma levels of leptin have been suggested as an independent risk factor for coronary artery disease $(10,11)$.

Curcumin is the orange-yellow pigment extracted from the famous spice turmeric. Curcumin is a unique phytochemical owing to its numerous molecular targets and diversity of biological activities. The efficacy of curcumin supplementation has been shown against a wide range of diseases including anxiety and depression $(12,13)$, osteoarthritis $(14,15)$, metabolic syndrome (16), dyslipidemia (17-19), atherosclerosis (20, 21), chronic complications due to sulfur mustard intoxication (22-25), solid tumors (26) and inflammation (27).

Interestingly, curcumin could modify almost all features of MetS (28). There is evidence indicating that curcumin lowers plasma levels of total cholesterol, LDL-cholesterol (LDL-C), triglyceride and glucose, and increases those of HDL-C (19, 29-33). Insulin-sensitizing (34-37), anti-obesity (38-40) and anti-hypertensive (41) effects of curcumin are other properties of this natural product reported in experimental studies. Experimental studies have also identified 
adiponectin and leptin as targets of curcumin $(42,43)$. However, clinical evidence on the impact of curcumin supplementation on circulating levels of these two adipokines has been scarce. Hence, this study aimed to evaluate changes in serum levels of adiponectin and leptin, and the ratio of these two adipokines, following curcumin supplementation in patients with metabolic syndrome. A secondary aim was to pool the results of clinical trials in order to estimate the effect size for the impact of curcumin on circulating adiponectin and leptin concentrations.

\section{Materials and methods}

\section{Subjects}

142 This study is a post-hoc analysis performed on the samples obtained from our previous 143 investigation (17). Participants were recruited from the Cardiology and Endocrinology Clinics of 144 the Baqiyatallah Hospital (Tehran, Iran). Inclusion criteria were males and females who were not originally receiving lipid-lowering therapy, for whom a diagnosis of MetS was made according to the criteria defined by the National Cholesterol Education Program Adult Treatment Panel III (NCEP-ATP III) guidelines as follows: $\geq 3$ of the following conditions: waist circumference $\geq$ $102 \mathrm{~cm}$ (male) or $\geq 88 \mathrm{~cm}$ (female), blood pressure $\geq 130 / 85 \mathrm{mmHg}$, triglycerides $\geq 1.7 \mathrm{mmol} / \mathrm{L}$, HDL-C $<1.03 \mathrm{mmol} / \mathrm{L}$ (males) or $<1.29 \mathrm{mmol} / \mathrm{L}$ (females), fasting blood glucose $\geq 6.1 \mathrm{mmol} / \mathrm{L}$ (44).

Exclusion criteria were pregnancy or breastfeeding, lack of compliance with the study medication (defined as not using the medication for $>1$ week), participation in a concomitant trial, hypersensitivity to the study medication, presence of malignancies and impossibility to give informed consent. The study protocol was given approval by the institutional Ethics Committee and written informed consent was obtained from participants.

\section{Study design}

158 This study was designed as a randomized double-blind placebo-controlled trial with a parallel159 group design. Subjects who met the inclusion criteria were randomly assigned to either curcumin 160 (Curcumin C3 Complex ${ }^{\circledR}$, Sami Labs LTD, Bangalore, India; $\mathrm{n}=59$ ) or matched placebo $(\mathrm{n}=$ 161 58) for a period of 8 weeks. Curcumin was administered at a daily dose of 1 g (500 mg b.i.d.) 162 based on the use of the same dose in our previous trial in obese individuals (45). In order to 163 improve the bioavailability of curcumin, 5 mg piperine (Bioperine ${ }^{\circledR}$; Sami Labs LTD, Bangalore, 
164

165

166

167

168

169

170

171

172

173

174

175

176

177

178

179

180

181

182

183

184

185

186

187

188

189

190

191

192

193

194

India) was added to each $500 \mathrm{mg}$ curcumin capsule (46). C3 Complex ${ }^{\circledR}$ preparation that was used in the present study contained three major curcuminoids i.e. curcumin, demethoxycurcumin and bisdemethoxycurcumin in a patented ratio. Placebo capsules contained the same amount of lactose plus $5 \mathrm{mg}$ piperine. This trial was registered at the UMIN Clinical Trials Registry (http://www.umin.ac.jp/ctr/) under Trial No. UMIN000018339.

\section{Blood Sampling}

Overnight fasting blood samples were collected at baseline and at study end. The samples were allowed to clot for about 30 minutes and then centrifuged at $750 \mathrm{~g}$ for 10 minutes to obtain serum. Sera were aliquoted and frozen at $-80^{\circ} \mathrm{C}$ until measurements.

\section{Measurements}

Serum concentrations of leptin and adiponectin were determined using the enzyme linked immunoassay technique with commercial kits. Weight, height, and systolic and diastolic blood pressures were measured according to standard procedures (47). BMI was calculated as weight in kilograms divided by height in meters squared $\left(\mathrm{m}^{2}\right)$.

\section{Statistical analysis}

Statistical analyses were performed using the SPSS software version 11.5 (SPSS Inc., Chicago, Illinois, USA). Data were expressed as mean \pm SD or number (\%). Within-group comparisons were performed using paired samples t-test (for normally distributed data) or Wilcoxon signedranks test (for non-normally distributed data). Between-group comparisons were performed using independent samples $t$-test (for normally distributed data) or Mann-Whitney $U$ test (for non-normally distributed data). Categorical variables were compared using Chi-square test. Bivariate correlations between changes in serum levels of leptin, adiponectin and leptin:adiponectin ratio were performed using Pearson's (for normally distributed data) and Spearman's (for non-normally distributed data) correlation coefficients. Univariate analysis of covariance (ANCOVA) using general linear model was used to adjust for the effect of potential confounders on the association between curcumin supplementation and changes in serum levels of adiponectin, leptin and leptin:adiponectin ratio. 


\section{Quantitative data synthesis}

Pooled analysis was performed using the Comprehensive Meta-Analysis (CMA) V2 software (Biostat, NJ) (48). Circulating adiponectin and leptin concentrations were collated in ng/mL. Standard deviations at one time point were calculated with the formula SD $=$ SEM $\times$ square root $n$ (SEM: standard error of the mean, $n$ : number of participants). Standard deviations (SDs) of the mean difference were calculated using the formula: square root $\left[\left(\mathrm{SD}_{\text {pre-treatment }}\right)^{2}+\left(\mathrm{SD}_{\text {post-treatment }}\right)^{2}\right.$ $\left.-\left(2 \mathrm{R} \times \mathrm{SD}_{\text {pre-treatment }} \times \mathrm{SD}_{\text {post-treatment }}\right)\right]$, assuming a correlation coefficient $(\mathrm{R})=0.5$. Net changes in measurements (change scores) were calculated as follows: (measure at end of follow-up in the treatment group - measure at baseline in the treatment group) - (measure at end of follow-up in the control group - measure at baseline in the control group). A random-effects model and the generic inverse variance method were used owing to the heterogeneity of studies in terms of design (parallel or cross-over), dosage and formulation of curcumin, and inter-study variations in the inclusion criteria (underlying disease, age, gender and anthropometric indices).

\section{Results}

One hundred and seventeen subjects met the inclusion criteria and were assigned to either curcumin $(n=59)$ or placebo $(n=58)$. One hundred subjects completed the trial. Nine subjects in the curcumin group and eight subjects in the placebo group did not complete the study due to loss to follow-up (Figure 1). The number of drop-outs was not different between the study groups.

Curcumin and placebo groups were comparable at baseline with respect to age, gender, smoking frequency, systolic blood pressure (SBP) and diastolic blood pressure (DBP). However, BMI ( $p$ $=0.002)$ and serum levels of glucose $(p<0.001)$ and HbA1c $(p=0.035)$ concentrations were higher in the curcumin group (Table 1). There was also no significant difference between the curcumin group and placebo group in terms of baseline serum adiponectin $(p=0.795)$ and leptin $(\mathrm{p}=0.292)$ concentrations and leptin:adiponectin ratio $(\mathrm{p}=0.526)$. Within-group analysis revealed a significant increase in serum adiponectin concentrations and reduction in leptin:adiponectin ratio in both curcumin $(\mathrm{p}<0.001)$ and placebo $(\mathrm{p}<0.001)$ groups. Serum leptin concentrations were reduced in the curcumin group $(\mathrm{p}<0.001)$ but did not change significantly in the placebo group $(\mathrm{p}=0.078)($ Table 2$)$. 
225 Between-group comparison of the change values revealed a significant elevation of serum 226 adiponectin $(\mathrm{p}<0.001)$ and a significant reduction of serum leptin concentrations $(\mathrm{p}<0.001)$ in the 227 curcumin compared with placebo group. Likewise, serum leptin:adiponectin ratio was 228 significantly reduced in the curcumin versus placebo group $(\mathrm{p}<0.001)$ (Table 2).

229 In order to check the effect of changes in serum lipids and glucose as potential confounders on 230 the observed changes in adipokines, univariate ANCOVA was performed. Assignment to 231 treatment group (yes/ no), and changes in serum levels of LDL-C, HDL-C, total cholesterol, 232 triglycerides, $\mathrm{Lp}(\mathrm{a})$ and glucose were separately entered into the model as independent variables. Another adjustment was also performed for baseline differences in BMI and serum concentrations of glucose and HbA1c. According to the results, the impact of curcumin supplementation on dependent variables including changes in serum concentrations of adiponectin $(p<0.001)$, leptin $(p=0.044)$ and leptin:adiponectin ratio $(p=0.001)$ remained statistically significant after adjustment for potential confounders. The impact of curcumin supplementation on the above-mentioned efficacy measures also remained significant after adjustment for baseline differences in BMI, serum glucose and HbA1c concentrations $(p<0.001)$. As reported in our previous report (17), curcumin were well-tolerated during the study. There were two reports of diarrhea, two reports of constipation, one report of headache, and two reports of skin rash in the curcumin group. Headache $(n=2)$ and constipation $(n=1)$ were reported adverse events in the placebo group. None of the drop-outs in this trial were due to the above244 mentioned adverse events.

\section{Bivariate correlations}

247 Changes in serum adiponectin concentrations were correlated with changes in HDL-C $(p=0.002)$,

248 whilst changes in serum leptin levels and serum leptin:adiponectin ratio were not found to be 249 associated with any of the assessed parameters. In the placebo group, there were significant

250 correlations between changes in serum adiponectin $(\mathrm{p}=0.010)$ and leptin:adiponectin ratio $251(\mathrm{p}=0.004)$ ratio with triglyceride changes. There was no significant correlation between changes 252 in serum adiponectin and leptin concentrations in either of the studied groups (Table 3). 
255 Meta-analysis of data from three RCTs (including the present study) using a random-effects 256 model showed that curcumin supplementation can increase plasma adiponectin (WMD: 76.78\%, 257 95\% CI: 6.14, 147.42; $p=0.0330$ ). With respect to plasma leptin concentrations, a reduction by 258 26.49\% was calculated (95\% CI: -70.44, 17.46), yet this effect size did not reach statistical 259 significance $(p=0.238)$ (Figure 2).

260

261

\section{Discussion}

262 The findings of this randomized controlled trial suggested a significant increase in serum levels of adiponectin and reduction in serum levels of leptin following 8 weeks of supplementation with 264 curcumin in patients with metabolic syndrome. To the best of author's knowledge, this is among 265 the very few studies dealing with the effect of curcumin on adipokines, and the first exploring 266 this issue in patients with metabolic syndrome. In a trial among subjects with prediabetes, 267 supplementation with curcumin (1500 mg/day) for 9 months was reported to increase plasma 268 adiponectin concentrations by 23.5\% (49). In another study in patients with type 2 diabetes, 6269 month supplementation with curcumin (1500 mg/day) reduced plasma leptin levels by 65\%, and 270 increased adiponectin by 152\% (50). In contrast to the above results, in another trial in patients 271 with major depressive disorder, 8-week supplementation with curcumin (1000 mg/day) was 272 found to increase plasma leptin levels by 23\%, though this increase did not reach statistical 273 significance (51).

274 Insulin resistance is defined as impairment of insulin action on glucose, lipid and protein 275 metabolism. It is closely associated with adipose tissue. Excessive visceral and subcutaneous 276 adipose tissue causes adipocyte dysfunction which can lead to inflammation through activation 277 of JNK and $\mathrm{NF}$ B. Inflammation causes impaired adipokine secretion reflected as decreased 278 adiponectin and increased leptin levels (52, 53). Adiponectin and leptin mediate insulin 279 sensitivity through AMPK (5’AMP-activated protein kinase) pathway. AMPK is a master switch 280 which controls energy status in the cell, and its activation leads to enhanced $\beta$-oxidation and reduced fatty acid esterification to triglycerides (54). Moreover, several studies have suggested that leptin:adiponectin ratio could serve as a useful index of insulin resistance and atherogenic 283 risk in both diabetic and non-diabetic populations (55-57). There are also reports showing the 284 association between leptin:adiponectin ratio and low-grade inflammation, carotid intima media 
thickness,. Arterial stiffness, first cardiovascular event and number of metabolic syndrome components $(58,59)$.

287

Some previous studies have revealed that curcumin could decrease insulin resistance by increasing fatty acid oxidation. Na et al. indicated that curcumin improves insulin resistance in skeletal muscles through activation of AMPK and fatty acid $\beta$-oxidation (60). These findings were approved in a later trial in diabetic type2 patients (61). Similarly, in an experimental study on C57BL/6J mice, it was indicated that curcumin can improve insulin resistance through inhibiting the expression of lipogenic genes and inflammation in the adipose tissue (39). In another experimental study, Weisberg et al. reported that curcumin ameliorates inflammation due to visceral adiposity, and this effect is accompanied by adiponectin elevation and mitigation of insulin sensitivity (40). There is also in vitro evidence indicating that curcumin blocks leptin signaling and prevents hyperlipidemia-induced oxidative stress, hepatic stellate cell activation, and liver fibrogenesis (62). The favorable impact of curcumin ssupplementation on adiponectin and leptin in this study is consistent with the reduction in serum glucose of the same individuals reproted previously (63). In our previous report, however, no significant effect could be detected in HbA1c which might be due to the short duration of follow-up.

301

302

303

304

305

306

307

308

309

310

311

312

313

314

315

Aside from insulin resistance, dyslipidemia is another prevalent feature of MetS, commonly presented as low HDL-C concentrations and elevated levels of triglycerides. This phenotype is referred to as atherogenic dyslipidemia, and is a promoter of insulin resistance. The beneficial effects of curcumin on lipid indices has been reported in our previous report from the same trial, indicating reductions in LDL-C, triglycerides and Lp(a) and elevations in HDL-C (17). This lipid-modifying effect of curcumin has also been reported in some other trials $(19,50,61)$.

As reported previously $(16,17)$, curcumin supplementation was safe in this trial. Curcumin has been approved by US FDA as a "generally recognized as safe" supplement, and its tolerability has been confirmed in several clinical studies. Therefore, owing to its safety and beneficial effects on several features of metabolic syndrome, curcumin may be suggested as a routine supplement for patients with metabolic syndrome.

Hitherto, several lines of evidence have suggested adiponectin as a key player in limiting the pathogenesis of obesity-related diseases including metabolic syndrome, non-alcoholic fatty liver disease and cardiovascular disease. The protective effects of adiponectin in reducing the risk of cardiometabolic diseases could be attributed to improvement of lipid and glucose metabolism as 
well as antioxidant, anti-inflammatory, anti-thrombotic, anti-hypertensive and antiatherosclerotic actions of this adipokine (64). These beneficial effects are mediated by the capacity of this adipokines to interact with important mediators/signaling molecules/pathways involved in cardiometabolic disturbances (65). Interestingly, curcumin has been shown to have the same multi-target capacity of action, and its capacity to interact with several key regulators such as transcription factors (eg. NFқB and activator protein 1), enzymes (cyclooxygenases, lipoxygenase and AMPK) pro-inflammatory cytokines, acute phase proteins, antioxidants, growth afctorrs, hormones, secondary messengers and nitric oxide, along with direct effects on adipokines production, could justify the beneficial cardiometabolic effects of this phytochemical (65). The present study was limited in a number of ways. First, this was a short-term trial and it 326 is unknown if longer durations of supplementation could cause further improvements in 327 circulating adiponectin and leptin concentrations. Second, this study tested the effects of a single 328 dose of curcumin, hence any dose-response association for the metabolic effects of curcumin 329 remains unclear. Finally, although circulating leptin, adiponectin and their ratio could serve as 330 indirect biomarkers of insulin resistance, insulin resistance was not measured in this study.

331 In conclusion, the present trial provided the first evidence on the improvement of circulating 332 adiponectin, leptin and leptin:adiponectin ratio in patients with metabolic syndrome. Future studies are encouraged to ascertain the impact of supplementation duration and curcumin dose on 334 the observed beneficial effects, and also the value of improving adipokine status with curcumin 335 in obese individuals and its plausible association with changes in body weight and fat content. 336 Finally, evaluation of the impact of curcumin on known measures of insulin resistance, including 337 hyperinsulinemic euglycemic clamp, homeostatic model assessment of insulin resistance 338 (HOMA-IR), and quantitative insulin sensitivity check index (QUICKI), is greatly 339 recommended.

\section{Acknowledgments}

342 This study was financially supported by Clinical Trial Research Center (Tehran, Iran) and Iran 343 National Science Foundation (INSF). The authors gratefully acknowledge Sami Labs LTD 344 (Bangalore, India) for providing the drug material used in this trial. 
347 Conflict of interest

348 Muhammed Majeed is the CEO of Sabinsa Corporation and Sami Labs Ltd.

349

350

351

352

353

354

355

356

357

358

359

360

361

362

363

364

365

366

367

368

369

370

371

372

373 


\section{References}

375 1. Reaven GM. Role of insulin resistance in human disease. Diabetes. 1988;37(12):1595-607.

376 2. Sahebkar A, Mohammadi A, Atabati A, Rahiman S, Tavallaie S, Iranshahi M, et al. Curcuminoids 377 Modulate Pro-Oxidant-Antioxidant Balance but not the Immune Response to Heat Shock Protein 27 and 378 Oxidized LDL in Obese Individuals. Phytotherapy Research. 2013;27(12):1883-8.

379 3. Azimi-Nezhad M, Herbeth B, Siest G, Dadé S, Ndiaye NC, Esmaily $H$, et al. High prevalence of metabolic syndrome in Iran in comparison with France: what are the components that explain this? Metabolic syndrome and related disorders. 2012;10(3):181-8.

4. Liu M, Liu F. Transcriptional and post-translational regulation of adiponectin. Biochem J. 2010;425:41-52.

5. Matsuzawa Y, Funahashi T, Kihara S, Shimomura I. Adiponectin and metabolic syndrome. Arteriosclerosis, thrombosis, and vascular biology. 2004;24(1):29-33.

386 6. Xydakis AM, Case CC, Jones PH, Hoogeveen RC, Liu M-Y, Smith EOB, et al. Adiponectin, inflammation, and the expression of the metabolic syndrome in obese individuals: the impact of rapid weight loss through caloric restriction. The Journal of Clinical Endocrinology \& Metabolism. 2004;89(6):2697-703.

3907 Lee $H$, Lee $M$, Joung $H$. Adiponectin represents an independent risk factor for hypertension in middle aged Korean women. Asia Pacific journal of clinical nutrition. 2007;16(1):10.

8. Lau DC, Dhillon B, Yan H, Szmitko PE, Verma S. Adipokines: molecular links between obesity and atheroslcerosis. American Journal of Physiology-Heart and Circulatory Physiology. 2005;288(5):H2031H41.

9. Hutley L, Prins JB. Fat as an endocrine organ: relationship to the metabolic syndrome. The American journal of the medical sciences. 2005;330(6):280-9.

10. Wallace AM, McMahon AD, Packard CJ, Kelly A, Shepherd J, Gaw A, et al. Plasma leptin and the risk of cardiovascular disease in the west of Scotland coronary prevention study (WOSCOPS). Circulation. 2001;104(25):3052-6.

402 11. Considine RV, Sinha MK, Heiman ML, Kriauciunas A, Stephens TW, Nyce MR, et al. Serum immunoreactive-leptin concentrations in normal-weight and obese humans. New England Journal of Medicine. 1996;334(5):292-5.

12. Panahi Y, Badeli R, Karami GR, Sahebkar A. Investigation of the Efficacy of Adjunctive Therapy with Bioavailability-Boosted Curcuminoids in Major Depressive Disorder. Phytotherapy Research. 2015;29(1):17-21.

13. Esmaily H, Sahebkar A, Iranshahi M, Ganjali S, Mohammadi A, Ferns G, et al. An investigation of the effects of curcumin on anxiety and depression in obese individuals: A randomized controlled trial. Chinese journal of integrative medicine. 2015;21(5):332-8.

14. Panahi Y, Alishiri GH, Parvin S, Sahebkar A. Mitigation of Systemic Oxidative Stress by Curcuminoids in Osteoarthritis: Results of a Randomized Controlled Trial. Journal of dietary supplements. 2015.

15. Panahi Y, Rahimnia AR, Sharafi M, Alishiri G, Saburi A, Sahebkar A. Curcuminoid Treatment for Knee Osteoarthritis: A Randomized Double-Blind Placebo-Controlled Trial. Phytotherapy Research. 2014;28(11):1625-31.

16. Panahi Y, Hosseini MS, Khalili N, Naimi E, Majeed M, Sahebkar A. Antioxidant and antiinflammatory effects of curcuminoid-piperine combination in subjects with metabolic syndrome: A randomized controlled trial and an updated meta-analysis. Clinical Nutrition. 2015.

418 17. Panahi Y, Khalili N, Hosseini MS, Abbasinazari M, Sahebkar A. Lipid-modifying effects of 419 adjunctive therapy with curcuminoids-piperine combination in patients with metabolic syndrome: 420 Results of a randomized controlled trial. Complementary therapies in medicine. 2014;22(5):851-7. 
18. Sahebkar A. Curcuminoids for the management of hypertriglyceridaemia. Nature Reviews Cardiology. 2014;11(2):123-.

423 19. Mohammadi A, Sahebkar A, Iranshahi M, Amini M, Khojasteh R, Ghayour-Mobarhan M, et al. 424 Effects of supplementation with curcuminoids on dyslipidemia in obese patients: a randomized crossover trial. Phytotherapy Research. 2013;27(3):374-9.

20. Sahebkar A. Dual effect of curcumin in preventing atherosclerosis: the potential role of prooxidant-antioxidant mechanisms. Natural product research. 2014 (ahead-of-print):1-2.

21. Sahebkar A. Molecular mechanisms for curcumin benefits against ischemic injury. Fertility and sterility. 2010;94(5):e75-e6.

22. Panahi Y, Ghanei M, Bashiri S, Hajihashemi A, Sahebkar A. Short-term Curcuminoid Supplementation for Chronic Pulmonary Complications due to Sulfur Mustard Intoxication: Positive Results of a Randomized Double-blind Placebo-controlled Trial. Drug research. 2014.

23. Panahi Y, Sahebkar A, Parvin S, Saadat A. A randomized controlled trial on the anti-inflammatory effects of curcumin in patients with chronic sulphur mustard-induced cutaneous complications. Annals of clinical biochemistry. 2012;49(6):580-8.

24. Panahi Y, Ghanei M, Hajhashemi A, Sahebkar A. Effects of Curcuminoids-Piperine Combination on Systemic Oxidative Stress, Clinical Symptoms and Quality of Life in Subjects with Chronic Pulmonary Complications Due to Sulfur Mustard: A Randomized Controlled Trial. Journal of dietary supplements. 2014.

25. Panahi Y, Sahebkar A, Amiri M, Davoudi SM, Beiraghdar F, Hoseininejad SL, et al. Improvement of sulphur mustard-induced chronic pruritus, quality of life and antioxidant status by curcumin: results of a randomised, double-blind, placebo-controlled trial. British Journal of Nutrition. 2012;108(07):12729.

26. Panahi Y, Saadat A, Beiraghdar F, Sahebkar A. Adjuvant Therapy with Bioavailability-Boosted Curcuminoids Suppresses Systemic Inflammation and Improves Quality of Life in Patients with Solid Tumors: A Randomized Double-Blind Placebo-Controlled Trial. Phytotherapy Research. 2014;28(10):1461-7.

27. Sahebkar A. Are Curcuminoids Effective C-Reactive Protein-Lowering Agents in Clinical Practice? Evidence from a Meta-Analysis. Phytotherapy Research. 2014;28(5):633-42.

28. Sahebkar A. Why it is necessary to translate curcumin into clinical practice for the prevention and treatment of metabolic syndrome? Biofactors. 2013;39(2):197-208.

29. Ramırez-Boscá A, Soler A, Carrion MA, Dıaz-Alperi J, Bernd A, Quintanilla C, et al. An hydroalcoholic extract of Curcuma longa lowers the apo B/apo A ratio: implications for atherogenesis prevention. Mechanisms of ageing and development. 2000;119(1):41-7.

30. Boscá AR, Soler A, Carrión-Gutiérrez MA, Mira DP, Zapata JP, Diaz-Alperi Jn, et al. An hydroalcoholic extract of Curcuma longa lowers the abnormally high values of human-plasma fibrinogen. Mechanisms of ageing and development. 2000;114(3):207-10.

31. Alwi I, Santoso T, Suyono S, Sutrisna B, Suyatna FD, Kresno SB, et al. The effect of curcumin on lipid level in patients with acute coronary syndrome. Acta Med Indones. 2008;40(4):201-10.

32. Baum L, Cheung SK, Mok VC, Lam LC, Leung VP, Hui E, et al. Curcumin effects on blood lipid profile in a 6-month human study. Pharmacological Research. 2007;56(6):509-14.

33. Soni K, Kuttan R. Effect of oral curcumin administration on serum peroxides and cholesterol levels in human volunteers. Indian journal of physiology and pharmacology. 1992;36:273-.

34. Seo KI, Choi MS, Jung UJ, Kim HJ, Yeo J, Jeon SM, et al. Effect of curcumin supplementation on blood glucose, plasma insulin, and glucose homeostasis related enzyme activities in diabetic $\mathrm{db} / \mathrm{db}$ mice. Molecular nutrition \& food research. 2008;52(9):995-1004. 
35. Pari L, Murugan P. Changes in glycoprotein components in streptozotocin-nicotinamide induced type 2 diabetes: influence of tetrahydrocurcumin from Curcuma longa. Plant foods for human nutrition. 2007;62(1):25-9.

470 36. Kuroda $M$, Mimaki $Y$, Nishiyama $T$, Mae $T$, Kishida $H$, Tsukagawa $M$, et al. Hypoglycemic effects of turmeric (Curcuma longa L. rhizomes) on genetically diabetic KK-Ay mice. Biol Pharm Bull. 2005;28(5):937-9.

473 37. Jang E-M, Choi M-S, Jung UJ, Kim M-J, Kim H-J, Jeon S-M, et al. Beneficial effects of curcumin on hyperlipidemia and insulin resistance in high-fat-fed hamsters. Metabolism. 2008;57(11):1576-83.

38. Ejaz A, Wu D, Kwan P, Meydani M. Curcumin inhibits adipogenesis in 3T3-L1 adipocytes and angiogenesis and obesity in C57/BL mice. The Journal of nutrition. 2009;139(5):919-25.

39. Shao W, Yu Z, Chiang Y, Yang Y, Chai T, Foltz W, et al. Curcumin prevents high fat diet induced insulin resistance and obesity via attenuating lipogenesis in liver and inflammatory pathway in adipocytes. PloS one. 2012;7(1):e28784.

40. Weisberg SP, Leibel R, Tortoriello DV. Dietary curcumin significantly improves obesity-associated inflammation and diabetes in mouse models of diabesity. Endocrinology. 2008;149(7):3549-58.

41. Nakmareong S, Kukongviriyapan U, Pakdeechote P, Donpunha W, Kukongviriyapan V, Kongyingyoes $\mathrm{B}$, et al. Antioxidant and vascular protective effects of curcumin and tetrahydrocurcumin in rats with L-NAME-induced hypertension. Naunyn-Schmiedeberg's archives of pharmacology. 2011;383(5):519-29.

42. Shehzad A, Ha T, Subhan F, Lee YS. New mechanisms and the anti-inflammatory role of curcumin in obesity and obesity-related metabolic diseases. European journal of nutrition. 2011;50(3):151-61. curcumin on the development of azoxymethane-induced colonic preneoplastic lesions in male C57BL/KsJ-db/db obese mice. Nutrition and cancer. 2012;64(1):72-9.

44. Cleeman J, Grundy S, Becker D, Clark L. Expert panel on detection, evaluation and treatment of high blood cholesterol in adults. Executive summary of the third report of the National Cholesterol Education Program (NCEP) Adult Treatment Panel (ATP III). Jama. 2001;285(19):2486-97.

45. Mohammadi A, Sahebkar A, Iranshahi M, Amini M, Khojasteh R, Ghayour-Mobarhan M, et al. Effects of supplementation with curcuminoids on dyslipidemia in obese patients: a randomized crossover trial. Phytotherapy research : PTR. 2013 Mar;27(3):374-9. PubMed PMID: 22610853.

46. Atal C, Dubey R, Singh J. Biochemical basis of enhanced drug bioavailability by piperine: evidence that piperine is a potent inhibitor of drug metabolism. Journal of Pharmacology and Experimental Therapeutics. 1985;232(1):258-62.

47. Asgary S, Sahebkar A, Afshani MR, Keshvari M, Haghjooyjavanmard S, Rafieian-Kopaei M. Clinical Evaluation of Blood Pressure Lowering, Endothelial Function Improving, Hypolipidemic and AntiInflammatory Effects of Pomegranate Juice in Hypertensive Subjects. Phytotherapy Research. 2014;28(2):193-9.

48. Borenstein M, Hedges L, Higgins J, Rothstein H. Comprehensive meta-analysis version 2. Englewood, NJ: Biostat. 2005;104.

49. Chuengsamarn S, Rattanamongkolgul S, Luechapudiporn R, Phisalaphong C, Jirawatnotai S. Curcumin extract for prevention of type 2 diabetes. Diabetes Care. 2012;35(11):2121-7.

50. Chuengsamarn S, Rattanamongkolgul S, Phonrat B, Tungtrongchitr R, Jirawatnotai S. Reduction of atherogenic risk in patients with type 2 diabetes by curcuminoid extract: a randomized controlled trial. The Journal of nutritional biochemistry. 2014;25(2):144-50.

51. Lopresti AL, Maes M, Meddens MJ, Maker GL, Arnoldussen E, Drummond PD. Curcumin and major depression: A randomised, double-blind, placebo-controlled trial investigating the potential of peripheral biomarkers to predict treatment response and antidepressant mechanisms of change. European Neuropsychopharmacology. 2015;25(1):38-50. 
52. Castro AVB, Kolka CM, Kim SP, Bergman RN. Obesity, insulin resistance and comorbidities? Mechanisms of association. Arquivos Brasileiros de Endocrinologia \& Metabologia. 2014;58(6):600-9.

53. Shoelson SE, Lee J, Goldfine AB. Inflammation and insulin resistance. Journal of Clinical Investigation. 2006;116(7):1793.

54. Vettor R, Milan G, Rossato M, Federspil G. Review article: adipocytokines and insulin resistance. Alimentary pharmacology \& therapeutics. 2005;22(s2):3-10.

55. Finucane FM, Luan J, Wareham NJ, Sharp SJ, O'Rahilly S, Balkau B, et al. Correlation of the leptin:adiponectin ratio with measures of insulin resistance in non-diabetic individuals. Diabetologia. 2009 Nov;52(11):2345-9. PubMed PMID: 19756488. Pubmed Central PMCID: 2759015.

56. Zaletel J, Barlovic DP, Prezelj J. Adiponectin-leptin ratio: a useful estimate of insulin resistance in patients with Type 2 diabetes. Journal of endocrinological investigation. 2010 Sep;33(8):514-8. PubMed PMID: 20142631.

57. Satoh N, Naruse M, Usui T, Tagami T, Suganami T, Yamada K, et al. Leptin-to-adiponectin ratio as a potential atherogenic index in obese type 2 diabetic patients. Diabetes Care. 2004 Oct;27(10):2488-90. PubMed PMID: 15451921.

58. Norata GD, Raselli S, Grigore L, Garlaschelli K, Dozio E, Magni P, et al. Leptin:adiponectin ratio is an independent predictor of intima media thickness of the common carotid artery. Stroke; a journal of cerebral circulation. 2007 Oct;38(10):2844-6. PubMed PMID: 17823381.

59. Kappelle PJ, Dullaart RP, van Beek AP, Hillege HL, Wolffenbuttel BH. The plasma leptin/adiponectin ratio predicts first cardiovascular event in men: a prospective nested case-control study. European journal of internal medicine. 2012 Dec;23(8):755-9. PubMed PMID: 22819464.

60. Na L-X, Zhang Y-L, Li Y, Liu L-Y, Li R, Kong T, et al. Curcumin improves insulin resistance in skeletal muscle of rats. Nutrition, Metabolism and Cardiovascular Diseases. 2011;21(7):526-33.

61. Na LX, Li Y, Pan HZ, Zhou XL, Sun DJ, Meng M, et al. Curcuminoids exert glucose-lowering effect in type 2 diabetes by decreasing serum free fatty acids: a double-blind, placebo-controlled trial. Molecular nutrition \& food research. 2013;57(9):1569-77.

62. Tang $\mathrm{Y}$, Zheng S, Chen A. Curcumin eliminates leptin's effects on hepatic stellate cell activation via interrupting leptin signaling. Endocrinology. 2009;150(7):3011-20.

63. Panahi Y, Hosseini MS, Khalili N, Naimi E, Majeed M, Sahebkar A. Antioxidant and antiinflammatory effects of curcuminoid-piperine combination in subjects with metabolic syndrome: A randomized controlled trial and an updated meta-analysis. Clinical nutrition. 2015 Dec;34(6):1101-8. PubMed PMID: 25618800.

64. Esfahani M, Movahedian A, Baranchi M, Goodarzi MT. Adiponectin: an adipokine with protective features against metabolic syndrome. Iranian journal of basic medical sciences. 2015 May;18(5):430-42. PubMed PMID: 26124928. Pubmed Central PMCID: 4475650.

65. Aggarwal BB, Sung B. Pharmacological basis for the role of curcumin in chronic diseases: an ageold spice with modern targets. Trends in pharmacological sciences. 2009 Feb;30(2):85-94. PubMed PMID: 19110321. 
$559 \quad$ Figure legends

560 Figure 1. Flow chart of the trial.

561

562 Figure 2. Forest plot detailing weighted mean difference and 95\% confidence intervals for the 563 impact of curcumin on circulating adiponectin (upper plot) and leptin (lower plot) 564 concentrations. The pooled effect size is shown as red diamond. There was significant pooled 565 effect of curcumin on adiponectin concentrations while for leptin meta-analysis (in which the red 566 diamond crosses the vertical line that corresponds to the value of zero), the pooled effect did not 567 reach statistical significance.

568

569

570

571

572

573

574

575

576

577

578

579

580

581

582

583

584 
586 Table 1. Baseline characteristics of study groups.

\begin{tabular}{lll}
\hline & Curcumin & Placebo \\
\hline Age (y) & $44.80 \pm 8.67$ & $43.46 \pm 9.70$ \\
Female & $23(46 \%)$ & $27(54 \%)$ \\
\hline Smoking & $12(24 \%)$ & $8(16 \%)$ \\
BMI (kg/m $\left.{ }^{2}\right)$ & $25.46 \pm 2.46$ & $22.80 \pm 5.37$ \\
\hline SBP (mmHg) & $135.56 \pm 13.16$ & $135.70 \pm 14.74$ \\
DBP (mmHg) & $88.34 \pm 7.81$ & $88.72 \pm 8.18$ \\
Hs-CRP (g/L) & $6.52 \pm 2.16$ & $7.10 \pm 1.80$ \\
Adiponectin (ng/mL) & $12.67 \pm 2.13$ & $12.78 \pm 2.19$ \\
\hline Leptin (ng/mL) & $22.02 \pm 2.93$ & $22.64 \pm 2.97$ \\
\hline Leptin:adiponectin & $1.77 \pm 0.32$ & $1.82 \pm 0.37$ \\
\hline LDL-C (mg/dL) & $190.46 \pm 20.05$ & $157.10 \pm 17.29$ \\
HDL-C (mg/dL) & $31.50 \pm 4.67$ & $35.48 \pm 6.54$ \\
\hline Total cholesterol (mg/dL)l & $220.29 \pm 37.72$ & $184.08 \pm 17.37$ \\
\hline Triglycerides (mg/dL) & $199.60 \pm 23.44$ & $185.64 \pm 38.49$ \\
\hline Lp(a) (mg/dL) & $82.00 \pm 7.35$ & $84.48 \pm 8.47$ \\
\hline Glucose (mg/dL) & $155.46 \pm 40.89$ & $136.98 \pm 52.40$ \\
\hline HbA1c (\%) & $6.69 \pm 1.44$ & $6.07 \pm 1.33$ \\
\hline
\end{tabular}

587 BMI: body mass index; SBP: systolic blood pressure; DBP: diastolic blood pressure; hs-CRP: 588 high-sensitivity C-reactive protein; LDL-C: low-density lipoprotein cholesterol; HDL-C: high589 density lipoprotein cholesterol; Lp(a): lipoprotein(a). 
Table 2. Changes in serum adipokines concentrations during the trial.

\begin{tabular}{|c|c|c|c|c|c|c|c|c|c|}
\hline & Curcumin & & & & Placebo & & & & \\
\hline & Before & After & Change & $p$-value ${ }^{\text {a }}$ & Before & After & Change & $p$-value ${ }^{a}$ & $p$-value \\
\hline Adiponectin (ng/mL) & $12.67 \pm 2.13$ & $21.28 \pm 4.40$ & $8.61 \pm 4.31$ & $<0.001$ & $12.78 \pm 2.19$ & $15.97 \pm 2.69$ & $3.19 \pm 3.36$ & $<0.001$ & $<0.001$ \\
\hline Leptin (ng/mL) & $22.02 \pm 2.93$ & $17.50 \pm 2.42$ & $-4.52 \pm 3.72$ & $<0.001$ & $22.64 \pm 2.97$ & $21.56 \pm 3.82$ & $-1.08 \pm 4.25$ & 0.078 & $<0.001$ \\
\hline Leptin:adiponectin & $1.77 \pm 0.32$ & $0.86 \pm 0.20$ & $-0.92 \pm 0.37$ & $<0.001$ & $1.82 \pm 0.37$ & $1.39 \pm 0.33$ & $-0.43 \pm 0.48$ & $<0.001$ & $<0.001$ \\
\hline
\end{tabular}

a: comparison of before vs. after values in each group; ${ }^{b}$ : comparison of changes between the study groups. 
Table 3. Bivariate correlations between changes in serum adiponectin, leptin and adiponectin:leptin ratio with serum lipids and glucose.

\begin{tabular}{|c|c|c|c|c|c|c|}
\hline \multicolumn{7}{|c|}{ Curcumin group } \\
\hline & \multicolumn{2}{|c|}{ Adiponectin } & \multicolumn{2}{|l|}{ Leptin } & \multicolumn{2}{|c|}{ Leptin:adiponectin } \\
\hline & $r$ & $p$ & $r$ & $p$ & $r$ & $p$ \\
\hline TC & 0.231 & 0.114 & -0.104 & 0.481 & -0.186 & 0.205 \\
\hline LDL-C & 0.298 & 0.036 & -0.008 & 0.958 & -0.220 & 0.124 \\
\hline HDL-C & 0.430 & 0.002 & -0.108 & 0.455 & -0.250 & 0.080 \\
\hline TG & 0.123 & 0.396 & 0.082 & 0.573 & 0.054 & 0.707 \\
\hline $\mathrm{Lp}(\mathrm{a})$ & 0.051 & 0.727 & -0.095 & 0.510 & 0.006 & 0.969 \\
\hline Glucose & -0.245 & 0.086 & 0.061 & 0.674 & 0.184 & 0.201 \\
\hline \multicolumn{7}{|c|}{ Placebo group } \\
\hline & \multicolumn{2}{|c|}{ Adiponectin } & \multicolumn{2}{|l|}{ Leptin } & \multicolumn{2}{|c|}{ Leptin:adiponectin } \\
\hline & $r$ & $p$ & $r$ & $p$ & $r$ & $p$ \\
\hline TC & 0.144 & 0.318 & 0.109 & 0.450 & -0.052 & 0.722 \\
\hline LDL-C & 0.022 & 0.881 & 0.007 & 0.962 & -0.009 & 0.951 \\
\hline HDL-C & 0.095 & 0.510 & -0.044 & 0.760 & -0.069 & 0.636 \\
\hline $\mathrm{TG}$ & -0.363 & 0.010 & .165 & 0.252 & 0.397 & 0.004 \\
\hline $\mathrm{Lp}(\mathrm{a})$ & 0.108 & 0.456 & -0.010 & 0.943 & -0.074 & 0.609 \\
\hline Glucose & 0.206 & 0.151 & 0.073 & 0.614 & -0.084 & 0.561 \\
\hline
\end{tabular}

Bivariate correlations were assessed using Pearson's (for normally distributed data) and Spearman's (for non-normally distributed data) correlation coefficients. TC: total cholesterol; LDL-C: low-density lipoprotein cholesterol; HDL-C: high-density lipoprotein cholesterol; TG: triglycerides; sdLDL: small-dense low-density lipoprotein; Lp(a): lipoprotein(a). 


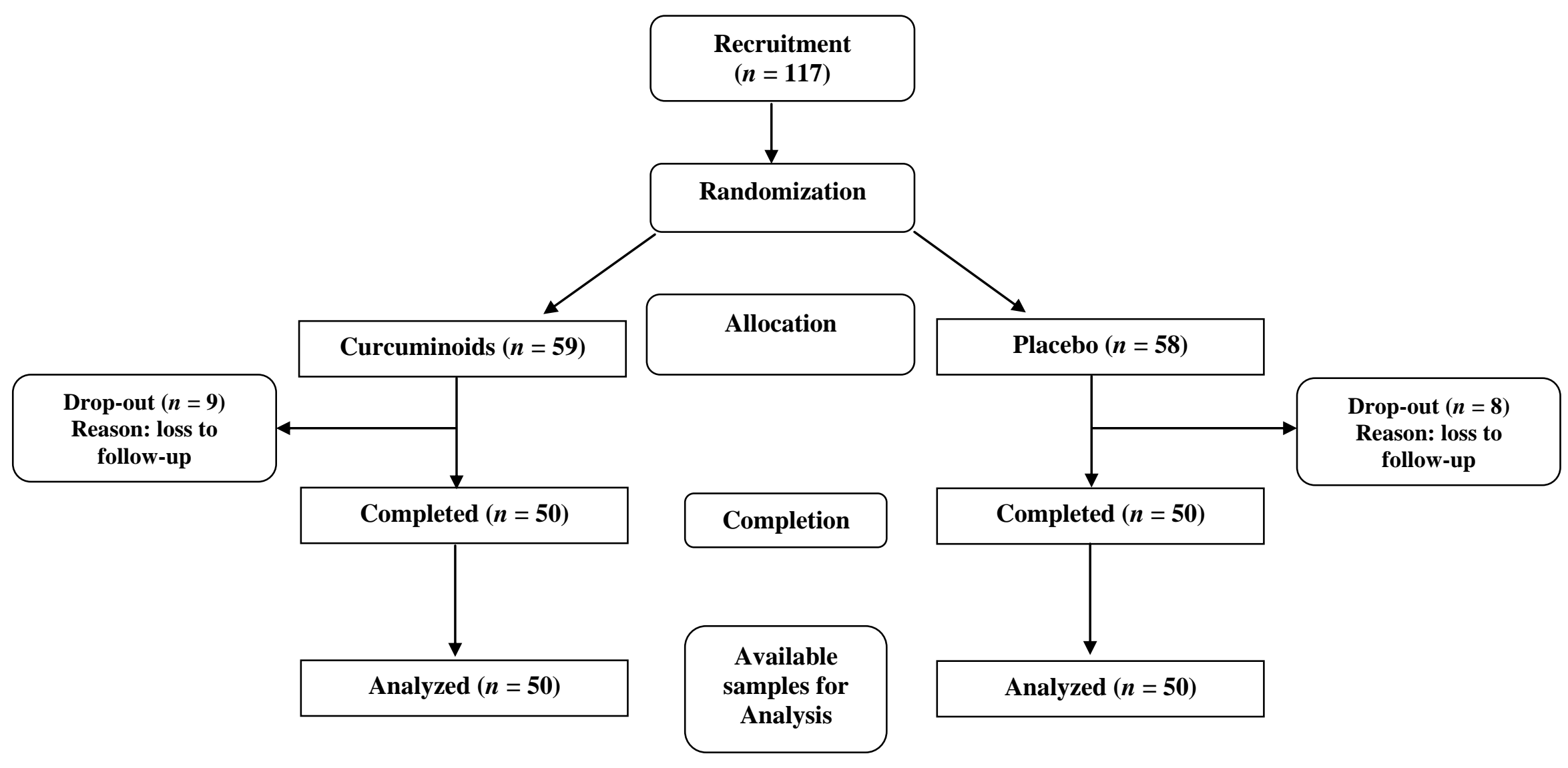


Study name

Chuengsamarn et al., 2012
Chuengsamarn et al., 2014
Panahi et al., 2015

\section{Study name}

Chuengsamarn et al., 2014

Panahi et al., 2015

Lopresti et al., 2015

\section{Statistics for each study}

Difference in means

23.480

165.130

43.000

76.781

tandard

error

8.197

10.364

5.062

36.040
Upper

Variance limit limit

67.185

107.411

25.619

1298.861

7.415

144.817

33.080

39.545

185.443

52.920

$6.145 \quad 147.418$
Z-Value

\subsection{5}

15.933

8.495

2.130

\begin{abstract}
p-Value
0.004

0.000

0.000

0.033
\end{abstract}

Difference in means and $95 \% \mathrm{CI}$
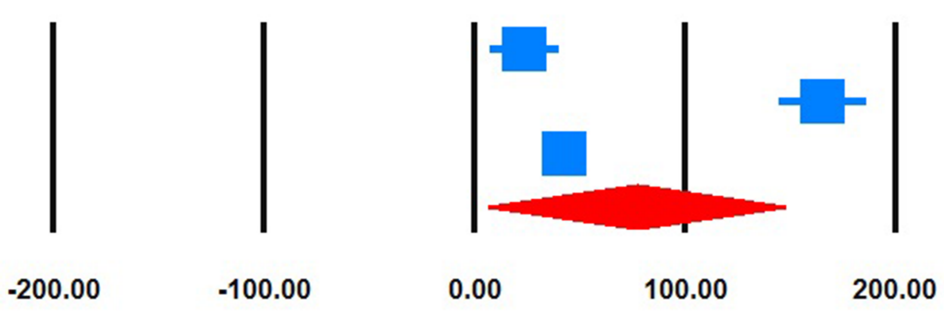

Favours Placebo Favours Curcumin

Statistics for each study

$\begin{array}{rrrrrrr}\begin{array}{c}\text { Difference } \\ \text { in means }\end{array} & \begin{array}{c}\text { Standard } \\ \text { error }\end{array} & \text { Variance } & \begin{array}{c}\text { Lower } \\ \text { limit }\end{array} & \begin{array}{c}\text { Upper } \\ \text { limit }\end{array} & \text { Z-Value } & \text { p-Value } \\ -68.230 & 9.080 & 82.451 & -86.027 & -50.433 & -7.514 & 0.000 \\ -15.760 & 2.780 & 7.731 & -21.210 & -10.310 & -5.668 & 0.000 \\ 22.760 & 30.296 & 917.866 & -36.620 & 82.140 & 0.751 & 0.453 \\ -26.488 & 22.425 & 502.864 & -70.439 & 17.464 & -1.181 & 0.238\end{array}$

Difference in means and $95 \% \mathrm{CI}$

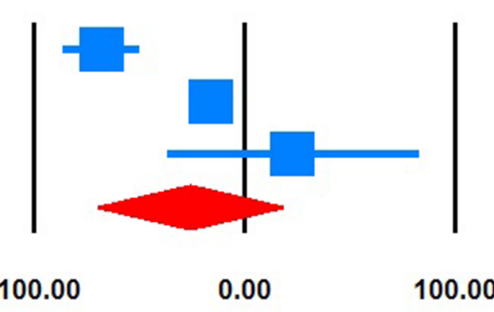

100.00

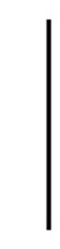

200.00

Favours Curcumin Favours Placebo 